ÉGYPTE

monde arabe

\section{Égypte/Monde arabe}

22 | 1995

Géographies de l'Égypte 1

\title{
L'urbanisation du monde arabe : un éclairage démographique
}

\section{Philippe Fargues}

\section{(2) OpenEdition}

\section{Journals}

Édition électronique

URL : https://journals.openedition.org/ema/599

DOI : $10.4000 /$ ema.599

ISSN : 2090-7273

\section{Éditeur}

CEDEJ - Centre d'études et de documentation économiques juridiques et sociales

\section{Édition imprimée}

Date de publication : 30 juin 1995

Pagination : 43-62

ISSN : 1110-5097

\section{Référence électronique}

Philippe Fargues, «L'urbanisation du monde arabe : un éclairage démographique », Égypte/Monde arabe [En ligne], 22 | 1995, mis en ligne le 08 juillet 2008, consulté le 07 juillet 2022. URL : http:// journals.openedition.org/ema/599; DOI : https://doi.org/10.4000/ema.599

Ce document a été généré automatiquement le 7 juillet 2022.

Tous droits réservés 


\title{
L'urbanisation du monde arabe : un éclairage démographique
}

\author{
Philippe Fargues
}

1 Les politiques restent préoccupés par la croissance urbaine : c'est ainsi que le thème « répartition de la population, urbanisation et migrations internes» occupait 25 des 399 paragraphes, soit $6 \%$, du Programme d'action de la Conférence internationale sur la population et le développement du Caire de septembre $1994^{1}$. Que Le Caire fût élu, neuf jours durant (du 5 au 13 septembre 1994), capitale de la démographie mondiale était d'ailleurs doublement emblématique: de la fixation sur la ville d'une peur démographique globale et de sa fixation particulière sur l'islam, perçu, sinon désigné, comme un frein de la transition, démographique. Le Caire est en effet à la fois l'une des agglomérations les plus denses du monde, bâtie sur l'espace le plus inextensible - la vallée du Nil -, et la ville d'al-Azhar, la plus haute institution de l'islam sunnite.

2 Le ton des politiques a cependant changé au fil du temps, à mesure qu'ils intégraient les résultats factuels de l'observation statistique et leur mise en perspective scientifique. L'urbanisation n'est plus le mal absolu qu'elle était apparue aux grands jours de l'exode rural : on rappelle volontiers qu'elle va dans le sens de l'histoire, qu'elle est à la fois produit et promoteur de la modernité, que l'agglomération des hommes est plus performante que leur dispersion ${ }^{2}$. Bref, la ville est à aménager, non à combattre.

3 L'urbanisation, en revanche, est un thème désormais boudé par les scientifiques de la population. Dernière en date de leur réunion planétaire, le congrès mondial de l'UlESP ${ }^{3}$ de Montréal (1993) faisait tenir ce thème dans une seule session, consacrée à la question précise des mégapoles; elle forme moins de $2 \%$ des actes de ce congrès, contre $14 \%$ de celui qui s'était tenu vingt ans plus tôt à Liège (1973). Une telle désaffection des démographes, en vingt ans seulement, confine au désaveu. Constater ce désaveu et essayer de le comprendre en examinant un cas particulier est un détour utile pour faire le point du phénomène lui-même dans une région particulière, en l'occurrence le monde arabe.

4 Dans une première partie, je répondrai à la question suivante: "Pourquoi les démographes se désintéressent-ils de l'urbanisation?» Plus précisément, je partirai 
d'une réponse évidente: c'est parce que le discours de la discipline ne s'est pas renouvelé sur ce sujet particulier. La croissance urbaine, envisagée comme modification progressive du rapport numérique entre populations urbaines et rurales, est en effet un phénomène de très longue durée, désormais bien décrit dans ses mécanismes strictement démographiques et pour la comptabilité duquel l'expérience passée des pays industriels paraît finalement préfigurer les tendances qui se dessinent dans les pays en développement. De plus, les rythmes du processus tendent un peu partout à se tasser, donnant un répit à l'analyste. De manière assez générale dans les pays en développement, un renversement est en effet survenu ces dernières années, l'urbanisation résultant de plus en plus de la croissance naturelle des populations urbaines et des échanges migratoires entre celles-ci, et de moins en moins de l'exode rural. Les dynamiques de l'ensemble urbain, d'un côté, et de l'ensemble rural, de l'autre, tendant à relever d'une même dynamique démographique globale simplement modulée par le degré d'agglomération des hommes -, l'étude spécifique des premières perd un peu sa raison d'être. Déjà contestée par les géographes, qui repèrent un continuum, une gradation de territoires que relient entre eux de multiples réseaux, par les économistes, qui soulignent les interdépendances, la dualité villes/ campagnes a désormais moins de pertinence pour le démographe lui-même.

Dans une seconde partie, je proposerai une question alternative : «Les modalités et les corrélats de la croissance urbaine, dans le monde arabe par exemple, ne donneraient-ils pas des motifs pour renouveler le discours démographique sur la ville? » En changeant d'échelle, en passant de l'observation globale des rapports de proportionnalité entre villes et campagnes à celle des processus démographiques qui agissent à l'intérieur d'une agglomération et sous-tendent sa croissance spatiale et ses nouvelles segmentations, on peut sans doute trouver quelques pistes sur lesquelles relancer ce thème.

L'urbanisation comme processus de concentration (la ville vue du dehors)

6 Sur la très longue durée - peut-être celle de toute l'histoire connue, une fois lissés les accidents de décrue démographique et de déconcentration spatiale -, la croissance de la population eut pour corollaire paradoxal la réduction de l'espace qu'elle occupe pour son habitat, sinon pour son économie. Ainsi, plus les hommes sont nombreux, plus ils se concentrent.

7 Les causes d'une telle concomitance sont complexes et, pour certaines, mal établies. Rappelons simplement l'une d'entre elles, parce qu'elle lie directement les deux phénomènes, croissance de la population et urbanisation, par une causalité allant de la seconde vers la première: il semble que le rapprochement spatial des hommes contribue à améliorer l'efficacité de leurs activités de production et d'échange ${ }^{4}$ et, par voie de conséquence, à repousser l'horizon du surpeuplement, c'est-à-dire à élever la capacité de croissance démographique. Dans le long terme, il n'y a donc sans doute pas lieu de s'alarmer de l'urbanisation. Dans le court terme, celui que l'action politique doit prendre en compte, un rythme élevé d'urbanisation peut cependant poser de délicats problèmes d'adaptation, moins aigus qu'au temps de l'exode rural en masse, mais amplifiés par la dimension des agglomérations d'aujourd'hui.

8 Comme sans doute la plupart des autres régions du monde, mais avec des décalages temporels, l'aire arabe aura connu une urbanisation en forme de courbe logistique: ascension d'abord lente et peut-être erratique, puis soutenue et de nouveau, tout récemment, en voie de ralentissement. On serait bien en peine, nous allons le voir, de 
distinguer l'expérience arabe d'un modèle planétaire général, sauf pour souligner quelques modalités locales de ce modèle, renvoyant soit à la géographie du système urbain, soit au rythme de sa construction.

Changements de forme du système urbain

9 Dans une région à l'intérieur de laquelle hommes, marchandises et capitaux circulent, les villes ne sont pas totalement indépendantes les unes des autres, mais évoluent en interaction: elles forment un système. Les systèmes dont il a été donné d'observer l'histoire longue, celui de l'Europe par exemple, possèdent des propriétés dynamiques propres, qui paraissent surdéterminer celles de chacune des villes qui composent le système. La trame que dessinent les villes semble ainsi relativement invariante dans le temps, de même que la hiérarchie de leurs effectifs de population : par delà les ruptures politiques ou économiques, le pouvoir et les acteurs économiques se relaient dans les mêmes sites ${ }^{5}$. Si l'on considère la carte des villes arabes et leur classement par taille, cette propriété dynamique ne s'applique pas : on observe au contraire deux moments d'instabilité, de repondération de certaines villes aux dépens des autres. Cela pourrait tenir à l'importance exceptionnelle des facteurs externes dans l'histoire économique et politique du monde arabe.

10 Durant une période très étendue, celle qui va de la conquête arabe jusqu'au XVI ${ }^{\mathrm{e}}$ siècle (découverte de la route du Cap), voire jusqu'au XvIII siècle (fin de l'économie-monde ottomane), les marches du désert abritent les plus grandes villes - d'est en ouest: Bagdad, Alep, Damas, le Caire, Fès, Marrakech - aux points précis de rupture de charge des caravanes. Une position stratégique de relais commercial entre des mondes extrêmement distants explique l'accumulation de richesses sur laquelle la ville se construit et l'installation du pouvoir politique qui la renforce, il est probable qu'elle n'exerce sur son environnement rural proche qu'une faible attraction migratoire et que l'urbanisation suive un rythme lent, marqué de discontinuités.

11 D'abord le détrônement du commerce terrestre par les voies maritimes, puis l'ouverture économique sur l'Europe, forcée ou librement choisie selon les pays, altérèrent une première fois les trames et hiérarchies urbaines, en revalorisant les littoraux : Alexandrie, Beyrouth, Tunis, Alger, Casablanca, Aden, ainsi qu'un semis de villes côtières de moindre importance, naquirent ou connurent un nouvel essor. La masse urbaine bascula vers le littoral, en parallèle ou en concurrence avec les anciennes villes de l'intérieur. Depuis cette époque, la croissance démographique des villes, côtières ou non, repose sur une combinaison de migrations de courte (l'exode rural) ou de moyenne (les migrations urbaines) distance, et de croissance naturelle, avec un dosage variable selon les villes et les périodes.

12 L'avènement de l'énergie pétrolière dans l'économie mondiale, combinée à la faiblesse de son utilisation par l'appareil productif régional, créa des systèmes rentiers et introduisit une seconde rupture dans la trame urbaine de l'Orient arabe, avec l'éclosion subite de grandes agglomérations sur les rives du Golfe et au cœur de la péninsule arabique. Le peuplement de souche, limité, ne suffit point à les alimenter, si bien qu'au moins dans sa phase initiale, c'est la migration de provenance lointaine qui fit l'essentiel de cette nouvelle urbanisation. Il fallut attendre la récession pétrolière des toutes dernières années pour que la croissance naturelle de ces populations urbaines, en retard sur le reste du monde arabe dans leur transition démographique, devienne là aussi le premier facteur d'urbanisation. 
13 En fait - et ce pourrait être la conclusion de ce court développement -, il n'y a pas, ou il n'y a plus, un système urbain arabe, mais des systèmes largement autonomes entre eux, qui sont ainsi passibles d'analyses séparées, à la rigueur comparatives. Le cadre des États est sans doute le plus adéquat, car les constructions nationales de ce siècle ont concrétisé la séparation d'économies qui n'échangent désormais presque plus entre elles et, en matière urbaine, elles ont consacré la suprématie des capitales autour desquelles l'État s'érigeait, et dont certaines étaient d'ailleurs un pur produit de la création nationale. Seul ensemble supranational à présenter toutefois l'allure d'un système, la région qui couvre la Péninsule arabique et le Levant est traversée par d'abondants flux migratoires qui instaurent un certain degré d'interdépendance, limité à des transferts financiers et, précisément, à la démographie urbaine.

Rythmes de l'urbanisation

14 Les mécanismes démographiques de la constitution des agglomérations dans les pays arabes n'ont rien d'exceptionnel. Au contraire, dans la succession des phases, ils auront suivi un schéma sans doute planétaire sur lequel il est superflu de s'appesantir, tant il a déjà été décrit. Au cours d'une première phase, qui s'acheva dans les années 70, la migration de provenance rurale forma l'essentiel de la croissance urbaine. Le rythme de celle-ci culmina vers 1960 : en moyenne 5,2 \% par an dans l'ensemble des pays arabes. Cependant, au fur et à mesure que grossissaient les effectifs des populations urbaines, leur excédent de croissance naturelle relayait l'exode rural comme premier facteur de croissance. L'urbanisation était entrée dans une seconde phase. Ce tournant passa longtemps inaperçu, alors que l'évolution des rapports entre villes et campagnes tendait au contraire à ralentir l'exode rural lui-même. D'un côté, en effet, la ville perdait une partie de son attrait, en raison notamment de la difficulté de s'y loger et de s'y employer, et de l'autre la campagne se diversifiait profondément sous l'influence de la ville: l'école et le centre de santé la pénétraient maintenant, tandis que le développement des transports périurbains accroissait son rayonnement en mettant la ville à portée quotidienne des ruraux.

Les migrations alternantes se substituèrent partiellement à l'installation en ville, si bien que la campagne cessa de se consacrer exclusivement à l'agriculture. On y exerça en proportion croissante des activités comparables à celles des villes. C'est ainsi que dans une couronne rurale distante de $50 \mathrm{~km}$ du Caire, les agriculteurs forment désormais moins du quart -dans certaines localités, moins du dixième-, de la population active. La baisse de la natalité récemment précipitée par la ville annonce sans doute la troisième phase, en cours dans les pays aujourd'hui développés, où la migration, interurbaine cette fois, devient à nouveau le premier facteur de la dynamique démographique des villes. Sans doute est-il utile de mentionner que, durant la succession de ces phases, la croissance démographique est restée déterminante de la croissance spatiale des agglomérations. C'est en tout cas ce que montre la surface bâtie $\mathrm{du}$ Grand Caire, zones industrielles ou commerciales incluses, dont la progression a suivi, dans un remarquable parallélisme, celle de la population sur toute la période qui va de 1917 à $1986^{6}$.

16 L'Arabie Saoudite, les émirats du Golfe et vraisemblablement la Libye ${ }^{7}$ sortent du schéma évoqué ci-dessus par un décalage à la fois spatial et temporel. La migration qui alimente leurs villes n'est pas, en effet, d'origine proche et rurale, mais de provenance lointaine et à prédominance urbaine, à l'exception des émigrés yéménites d'Arabie, près d'un million de personnes venues des campagnes - d'ailleurs pour la plupart 
retournées dans leur pays d'origine après la guerre du Golfe en 1991. En outre, elle apporte une contribution prépondérante à la croissance urbaine plus tardivement qu'ailleurs, jusqu'au milieu des années 80 , c'est-à-dire tant qu'une rente surabondante permet la réalisation d'ambitieux projets de construction. La construction démographique des villes du pétrole, de véritables cités-États dans le cas des petites principautés, procède non de la maturation des rapports entre économie et démographie locales, mais de la rencontre opportune d'une conjoncture économique planétaire (la demande de pétrole) avec une conjoncture politique régionale (l'insécurité des guerres qui valorise le pétrole) et avec la démographie de pays voisins (Levant, Yémen) ou relativement éloignés (Égypte, Turquie, Inde, Pakistan et Sud-Est asiatique).

Qu'ils soient sortis du schéma général (monarchies pétrolières) ou qu'ils l'aient suivi (autres pays), les pays arabes présentent des niveaux et des rythmes d'urbanisation très variés qui font - et ce sera ma seconde conclusion partielle - que le démographe ne saurait repérer un modèle arabe de l'urbanisation, mais une diversité de situations. Qu'on en juge aux quelques chiffres suivants ${ }^{8}$. L'étagement des niveaux, d'abord, couvre pratiquement le spectre mondial: du Soudan et du Yémen, où moins du tiers des habitants résident en ville (respectivement 24 et $33 \%$ en 1994) aux principautés du Golfe et à la bande de Gaza, qui comptent plus de $90 \%$ de citadins, on passe par une masse de pays où la proportion de population urbaine tourne autour de $50 \%$. Dans l'ensemble arabe, c'est une courte majorité citadine que l'on enregistre aujourd'hui (115 millions sur 225). Les rythmes de la croissance urbaine du demi-siècle écoulé, ensuite, révèlent la variété des positions de départ et des modalités du changement: pour 100 citadins en 1950, on en compte aujourd'hui moins de 500 dans les pays de vieille tradition citadine, comme l'Égypte, la Tunisie ou le Maroc, mais plus de 5.000 dans les pays où la poussée urbaine est la plus récente, et où se retrouvent pêle-mêle les plus pauvres (Mauritanie) et les plus riches (principautés du Golfe). Dans l'ensemble arabe, la montée urbaine de ce siècle est cependant d'une vigueur qui vaut d'être rappelée : 3,5 millions de citadins en 1900, 115 aujourd'hui. L'équilibre des systèmes urbains nationaux, enfin, révèle non seulement l'hétérogénéité arabe, mais le cloisonnement interne de ce monde : si les agglomérations de plus de 500.000 habitants (ou la capitale, dans les pays où de telles agglomérations n'existent pas) forment de moins de $15 \%$ (Algérie) à plus de $75 \%$ (Tunisie, Liban, Irak, Arabie, principautés du Golfe, Mauritanie) de la population urbaine, c'est un peu le résultat de l'enfermement des mouvements migratoires par les constructions économiques et administratives des États nationaux.

Regarder la ville du dedans

18 L'identité arabe qu'une vision globale de l'urbanisation ne révèle pas, peut-on la trouver dans des dynamiques internes à la ville, c'est-à-dire à un niveau organique?

Le passage à la ville, catalyseur des transitions

19 La transition démographique semble être un processus universel, mais elle ne se situe pas au-dessus des sociétés : d'une part, elle accompagne d'autres transitions et, d'autre part, elle épouse les aspérités de chaque terrain sociologique particulier ${ }^{9}$. Dans les sociétés arabes, ainsi peut-être que dans d'autres sociétés, trois traits pourraient caractériser cette interdépendance de la démographie et de la sociologie. En premier lieu, les moments et les rythmes de la transition démographique portent la marque de la persistance, jusqu'à récemment, d'un ordre patriarcal fort dans la famille et d'un 
ordre «néo-patriarcal ${ }^{10}$ dans la société et les systèmes politiques. Très schématiquement, cet ordre se distingue par la domination des hommes sur les femmes et, pour chaque sexe, des vieux sur les jeunes. En second lieu, la manière dont la transition démographique s'est déroulée, en particulier le rôle qu'a tenu la diffusion de l'instruction scolaire, aboutit à l'érosion - sinon à l'abolition - de cet ordre patriarcal : par certains aspects de leur statut, les femmes tendent à rejoindre les hommes et les jeunes à dépasser les vieux. En troisième lieu enfin, la résistance des valeurs et des structures héritées de l'ordre ancien crée un contexte propice à une crise de société, dont les expressions politiques peuvent revêtir diverses formes, de la mobilisation associative à la mobilisation violente. La ville est le lieu par excellence où commence, puis d'où se diffuse cette triple transition, à la fois démographique, sociologique et politique.

20 L'influence de l'ordre patriarcal sur la démographie ressort bien de l'examen des niveaux de la fécondité dans le monde arabe, qui présentent aujourd'hui leur dispersion maximum. Le déclin de la fécondité, comme d'autres changements sociaux, apparaît d'abord dans un sous-groupe limité de la population, une "élite » à partir de laquelle il gagne progressivement le gros de la population, au fur et à mesure que se diffusent les valeurs et les comportements de cette élite. A l'instar des autres sociétés, ces valeurs et comportements, «facteurs » de la baisse de la fécondité, sont liés à la fois à l'évolution des positions socio-économiques et à celle des rôles familiaux. Mais, à l'inverse de l'expérience des autres ensembles supranationaux, les facteurs qui expliquent les variations de la fécondité à l'intérieur des populations nationales perdent leur pouvoir explicatif lorsque l'on considère les différences entre sociétés nationales. C'est ici, je crois, qu'interviennent les structures patriarcales. Afin d'illustrer cette hypothèse, j'évoquerai rapidement deux facteurs essentiels : la richesse et l'éducation des femmes.

21 Dans presque tous les pays en développement, l'élévation du niveau de vie s'est accompagnée d'une réduction de la fécondité. Dans chaque pays arabe, de même, les plus basses fécondités s'observent dans les classes moyennes ou supérieures des villes, et les plus hautes dans les classes pauvres, paysannes notamment. Lorsque l'on compare les pays entre eux, la relation disparaît curieusement. Les fécondités moyennes les plus basses sont celles des pays à revenu national relativement bas ou moyen (Liban, Tunisie, Maroc, Égypte) et les plus hautes celles des pays les plus riches (Péninsule) ${ }^{11}$. Les particularités du mode d'accumulation des richesses et les modalités sociales et politiques de leur redistribution me paraissent éclairer cette inversion. Les économies politiques des pays arabes balancent en effet entre deux pôles, l'un productif et l'autre rentier. Le premier active les leviers "classiques » de la baisse de fécondité, notamment dans les villes : coût de l'enfant et exercice croissant de l'activité féminine rémunérée en dehors du foyer familial. Le second les inhibe au contraire : prenant en charge les coûts de l'enfant et organisant l'importation d'une main-d'œuvre féminine étrangère qui se substitue à une main-d'œuvre féminine nationale, les États rentiers de la Péninsule ont fourni les moyens de préserver la famille nombreuse et la femme au foyer. Ils ont ainsi donné un répit à l'ordre patriarcal de la famille, en utilisant les canaux redistributifs du système néo-patriarcal (clientèle).

En réalité, il n'y a pas deux types bien séparés, mais un continuum qui va de l'un à l'autre : chaque État arabe, à un titre ou à un autre, émarge à la rente pétrolière ${ }^{12}$ et possède des moyens, plus ou moins importants selon sa position dans la chaîne de 
circulation de la rente, de distribuer plus que le pays ne produit. C'est ainsi que les deux modèles de fécondité coexistent, avec un poids différent selon les pays et les périodes. L'expérience égyptienne est éloquente : après une baisse sous la présidence de Nasser, la fécondité se releva considérablement de 1973 à 1988. Cette période avait été celle de l'ouverture, notamment à l'émigration, et des transferts financiers en provenance du Golfe et des États-Unis. Tout se déroula comme si un regain de .confort économique avait permis aux familles de procréer à nouveau plus d'enfants et de garder les femmes au domicile conjugal ${ }^{13}$, c'est-à-dire de revigorer un ordre patriarcal que le nassérisme n'avait pas altéré en profondeur.

Plus active encore que le niveau de vie, l'éducation des femmes est un facteur important de la baisse de la fécondité. Cette caractéristique commune aux expériences contemporaines des pays en développement se retrouve bien à l'intérieur de chaque pays arabe. En Tunisie par exemple, les femmes d'éducation universitaire procréent en moyenne 2,2 enfants et les illettrées 5,0, avec une gradation régulière des unes aux autres. Concentrant les populations les plus instruites, la ville est donc, par ce canal également, le lieu de la baisse de fécondité. Cependant, comme c'était le cas du niveau de vie, le niveau d'instruction des femmes ne permet pas de classer les pays selon leur fécondité moyenne. Par exemple, plus instruite et plus urbaine que le Maroc, la Jordanie est aussi plus féconde, alors qu'on attendrait l'inverse. La raison en est que l'action de l'éducation des femmes sur le contrôle des naissances est modulée par le statut que la famille et la société leur offrent à la sortie de l'école, notamment par l'arbitrage entre mariage et exercice d'une activité économique.

Gaza en fournit le plus éloquent des exemples. Contrairement à tout ce que laisseraient attendre un taux d'urbanisation extrêmement élevé (97\%) et une durée moyenne de scolarité féminine plus longue que dans tous les autres pays arabes $(9,0$ ans chez les femmes âgées de 30 ans en 1992, c'est-à-dire à l'âge moyen de procréation), Gaza possède la natalité la plus haute du monde : un taux brut de natalité de 56,5 p. 1.000 en 1992, correspondant à une fécondité totale comprise entre 8 et 9 enfants par femme. Divers facteurs liés aux conditions de l'occupation militaire israélienne sont en cause, parmi lesquels un marché du travail déficitaire pour les hommes et pratiquement fermé aux femmes (5 \% sont actives entre 15 et 50 ans). Par ailleurs, si l'hyperfertilité est à juste titre interprétée comme l'une des meilleures armes des Palestiniens, puisqu'elle modifie rapidement les rapports numériques en leur faveur, elle présente la particularité d'être une arme en accord parfait avec l'ordre patriarcal, comme s'il existait une division du travail militant entre les sexes: les hommes dans l'arène publique du combat et les femmes dans la sphère privée de la procréation.

Pour illustrer maintenant la manière dont la transition démographique s'accompagne d'une remise en cause de l'ordre patriarcal, je me limiterai ici à observer comment l'instruction scolaire, facteur central de cette transition, perturbe, en se propageant dans la société, les hiérarchies ancestrales des sexes et des générations.

Avant la diffusion de l'école, c'est-à-dire jusqu'aux générations nées durant le premier tiers ou la première moitié de ce siècle, selon les pays, les rares établissements scolaires étaient réservés à une classe très minoritaire de la population. Qu'ils fussent en pratique réservés aux seuls garçons de cette classe aboutissait à produire une élite presque entièrement masculine, mais, pour l'immense majorité qui n'y avait pas accès, les deux sexes étaient égaux. Lorsque l'école se développa, d'abord en ville puis à la campagne, les familles y envoyèrent plus volontiers leurs garçons que leurs filles. Une 
nouvelle inégalité était née, qui allait concerner la masse de la population. C'est dans les générations 1950-60, aujourd'hui en âge d'exercer le pouvoir dans la famille et dans la société, que cette inégalité est la plus accusée. Au regard de ce simple facteur, l'ordre patriarcal est donc plus fort que jamais. Cependant, continuant son chemin, l'école s'ouvrit aux filles : dans les villes en tout cas, les nouvelles générations sont d'ores et déjà presque égales devant l'instruction primaire et le seront bientôt devant l'instruction secondaire. C'est lorsqu'elles atteindront l'âge des responsabilités familiales ou du pouvoir politique que les valeurs égalitaires portées par leur expérience achèveront de détrôner l'ordre ancien.

Le développement de l'instruction scolaire eut pour second effet d'inverser la hiérarchie des classes d'âge. Tandis que le modèle patriarcal plaçait les aînés au sommet, l'école donna la prééminence aux jeunes, sans cesse plus scolarisés que ne l'avaient été leurs pères. Pour la simple raison que l'école ne s'adresse qu'aux enfants, sa diffusion bientôt massive creusa une distance avec leurs parents : cette distance culmine dans les générations 1960-70. C'est ainsi que la jeunesse arabe passe à présent par un point critique où, par son savoir, elle dépasse plus que jamais ses aînés, qui conservent pourtant les principales positions d'autorité dans la famille et dans la société. En Égypte, mais aussi sans doute ailleurs, la rupture générationnelle est plus prononcée chez les femmes que chez les hommes, scolarisés depuis longtemps, et subit, lorsque l'on passe des campagnes aux villes, un déplacement vers le haut: inégalité devant la simple capacité à lire et à écrire dans les premières, mais devant les enseignements secondaires et universitaires dans les secondes. Le potentiel de contestation de l'ordre familial et social que cette rupture porte, devrait donc lui-même être plus fort chez les femmes et dans les villes que chez les hommes et dans les campagnes.

Je ne m'attarderai pas beaucoup sur le troisième volet de cette démographie organique: les formes que prend la contestation des hiérarchies héritées de l'ordre patriarcal. Tout d'abord parce que cette contestation relève surtout du politique, et son étude d'une autre discipline que la mienne. Ensuite parce que l'on dispose en la matière de beaucoup de professions de foi, mais de fort peu d'évidences scientifiques. Les professions de foi, portées par les médias et par quelques scientifiques, font de la mobilisation violente une sorte d'attribut fatal d'une croissance urbaine décrite comme un processus anarchique. Ce n'est pas ce que révèle la géographie de la violence politique en Égypte depuis la mi-1992. Admettant que celle-ci est une réponse, parmi d'autres possibles, à un ensemble de frustrations collectives ou simplement partagées par un grand nombre d'individus, j'ai examiné les corrélations spatiales entre la fréquence des actes de violence politique d'un côté et, de l'autre, un ensemble de facteurs de frustration potentielle, liés de près ou de loin à la démographie. Il en ressort que l'action violente ne présente pas de corrélation avec les causes qu'on lui attribue hâtivement - niveau de sous-emploi, degré de pauvreté, densité de l'habitat, taux d'urbanisation -, mais avec des facteurs qui relèvent plutôt de la sociologie et de l'anthropologie : le taux de violence s'accroît avec la fréquence des structures familiales polynucléaires, avec les indicateurs de dépendance du statut de la femme, avec l'acuité de la rupture générationnelle introduite par l'instruction scolaire. En bref, il y a violence là où persistent les traces d'ordre patriarcal que la modernité malmène.

Nouvelle segmentation ou lissage de l'espace social des villes? 
29 Paradoxalement, alors que, leur nombre s'accroissant, les hommes occupent une surface de moins en moins vaste du territoire, à l'intérieur du territoire occupé ils tendent à s'étaler, c'est-à-dire à uniformiser leur répartition spatiale ${ }^{14}$. La concentration de la population que l'on peut voir d'en haut, à petite échelle, laisse en effet place, dans une observation à grande échelle, à la déconcentration des zones les plus densément peuplées. Le desserrement de l'habitat urbain, tel qu'on peut l'observer depuis la fin des années 50 dans un pays comme la France où la croissance naturelle est basse, n'est encore qu'une tendance en train de se dessiner dans le monde arabe. En effet, héritage d'un passé tout récent de haute fécondité, une croissance naturelle encore élevée surcompense, au point de les masquer, les migrations centrifuges qui affectent désormais d'anciens noyaux urbains. Mais le fait nouveau est déjà là : les zones centrales de grandes agglomérations arabes sont désormais devenues émettrices de flux migratoires à destination de leurs périphéries. Lorsque l'on considère deux cartes du Très Grand Caire ${ }^{15}$ découpé en ses 608 unités censitaires, celle des densités de population en 1986 (dernier recensement) et celle des soldes migratoires entre 1976 et $1986^{16}$, une corrélation très fortement négative se dégage : dès cette période, $46 \%$ (282) des unités de l'agglomération, parmi les plus centrales et les plus denses, avaient un taux de migration nette négative parce qu'elles alimentaient l'urbanisation d'une couronne située hors des limites administratives de la ville. L'ensemble du Très Grand Caire lui-même n'avait plus qu'un taux de migration nette de 0,7 \% par an. Le prochain recensement étant prévu en 1996, il faudra attendre quelques années pour connaître les migrations de la décennie écoulée. Cependant, la poursuite de l'urbanisation périphérique ne fait pas de doute, de même que le déclin de la croissance naturelle de la population urbaine. Il est ainsi probable que le centre de l'agglomération du Caire soit désormais en cours de dépopulation. La même évolution caractérise vraisemblablement beaucoup d'autres agglomérations arabes. 
Figure 1 : Densités de population au recensement de 1986. Très Grand Caire

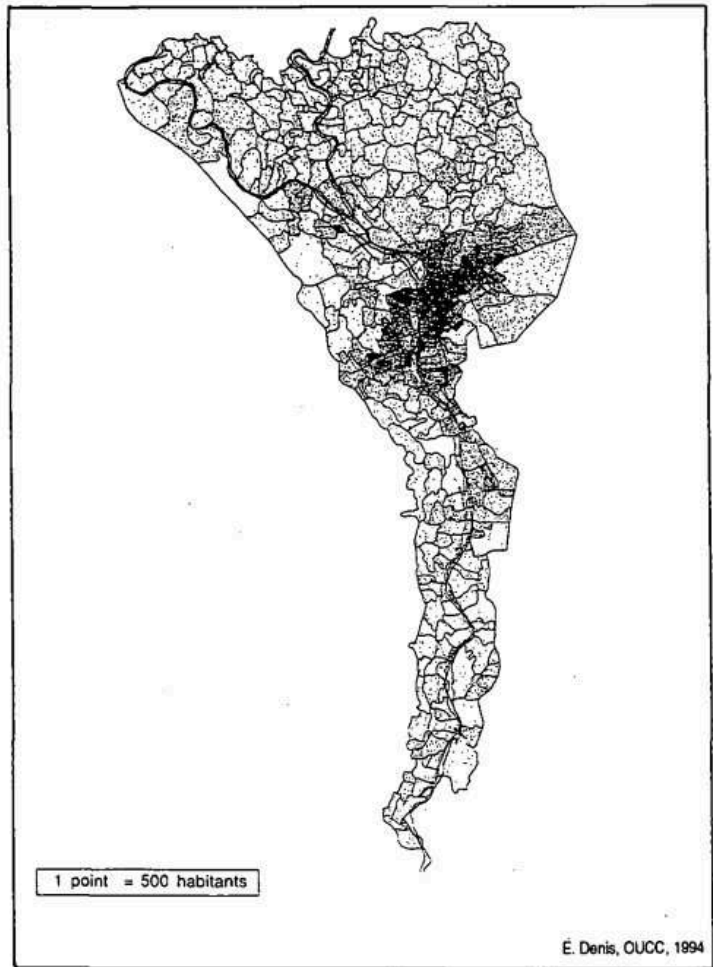

Figure 2 : Taux annuel de migration nette 1976-1986. Très Grand Caire

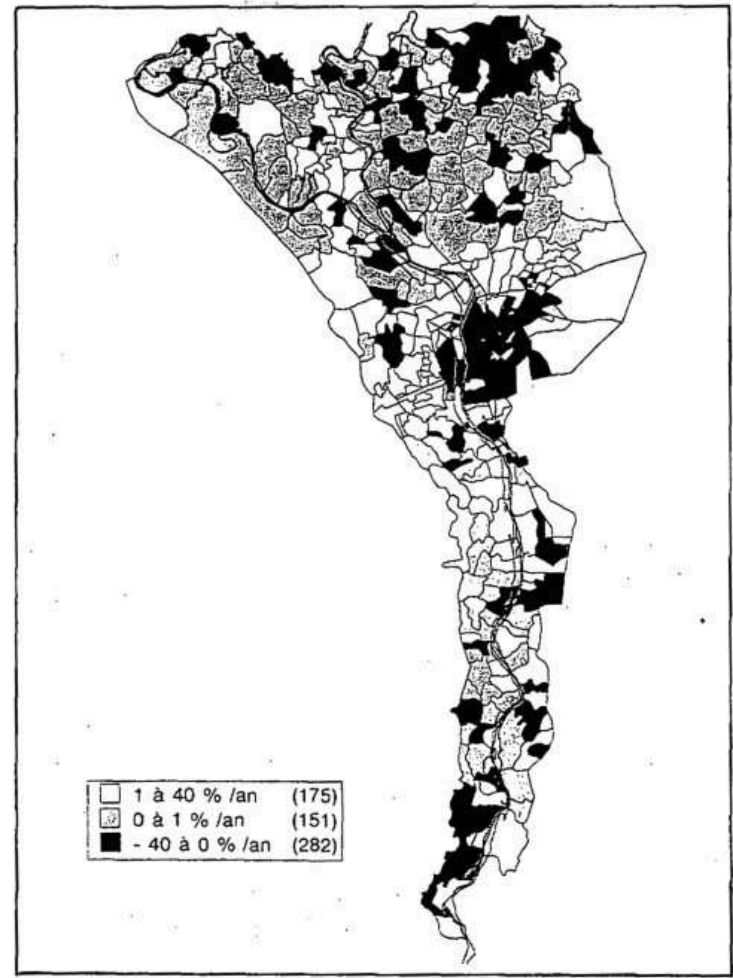


Figure 3 : Age moyen au recensement de 1986. Très Grand Caire

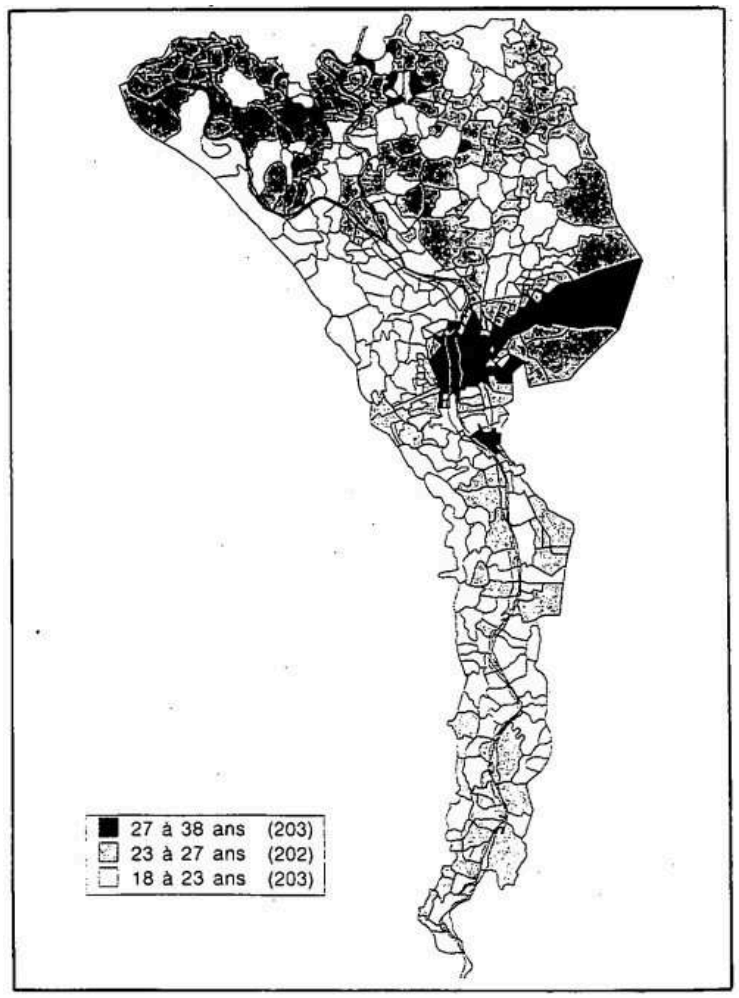

Laissant aux géographes l'analyse de ces nouvelles migrations intra-urbaines, le démographe s'intéressera aux liens qu'elles entretiennent avec les structures de la population, en particulier avec sa répartition en familles et en classes d'âge. La transition démographique n'est pas seulement la réduction de la fécondité. Beaucoup plus ancienne est sa première phase, le déclin de la mortalité, perceptible au Caire dès la tenue régulière d'une statistique des décès au début du siècle, mais s'accélérant à partir des années 50. Au fur et à mesure que la durée de vie s'allonge, les structures horizontales et verticales des familles se transforment. D'une part, les enfants sont plus nombreux à survivre à l'âge adulte et, d'autre part, la coexistence des générations s'allonge. Autrefois, un adulte avait perdu plusieurs frères et sœurs dans l'enfance. Par ailleurs, il était rare qu'il ait encore ses deux parents en vie. Aujourd'hui la fratrie entière a plus de chances de survivre et la proportion des familles où se côtoient trois, voire quatre générations ne cesse d'augmenter. Ainsi, alors qu'auparavant on avait de bonnes chances de succéder, dès son mariage ou peu après, à ses parents dans le domicile familial, on doit aujourd'hui "décohabiter». On se marie peut-être un peu plus tard qu'autrefois, mais de quelques années seulement, si bien que le déroulement du cycle familial suppose l'extension du parc de logements. Le centre étant saturé, c'est de plus en plus loin que l'on construit cet habitat de décohabitation. Voilà notamment pourquoi l'âge moyen de la population diminue par cercles concentriques lorsque l'on passe du centre aux périphéries. 
Figure 4 : Nombre moyen d'années d'instruction au recensement de 1986. Très Grand Caire

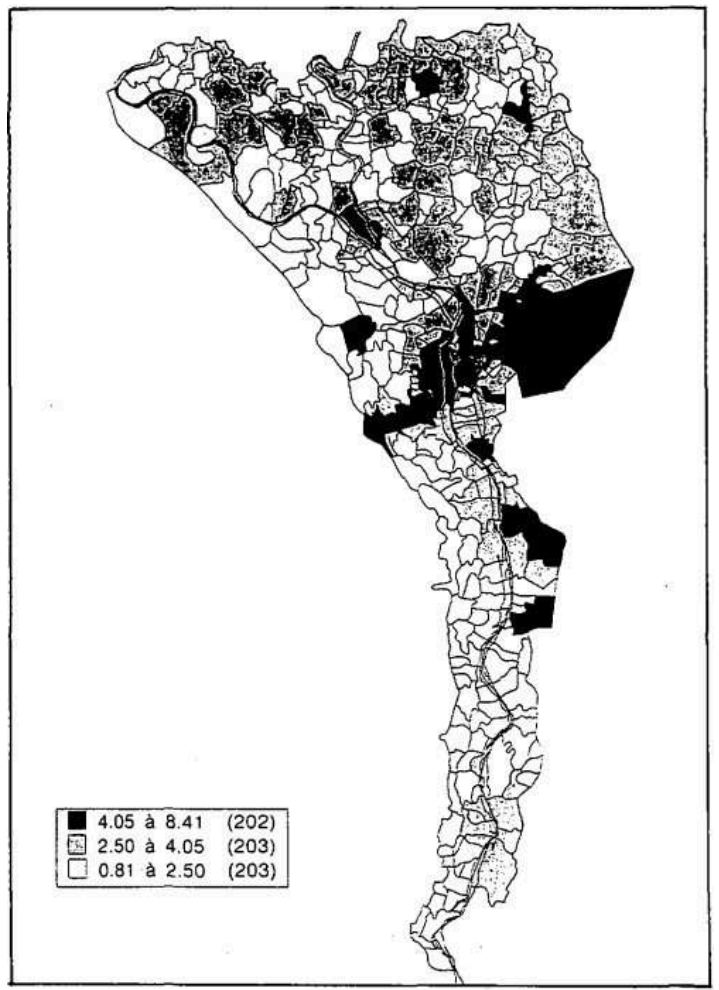

Figure 5 : Elévation du niveau d'instruction entre 1976 et 1986. Très Grand Caire.

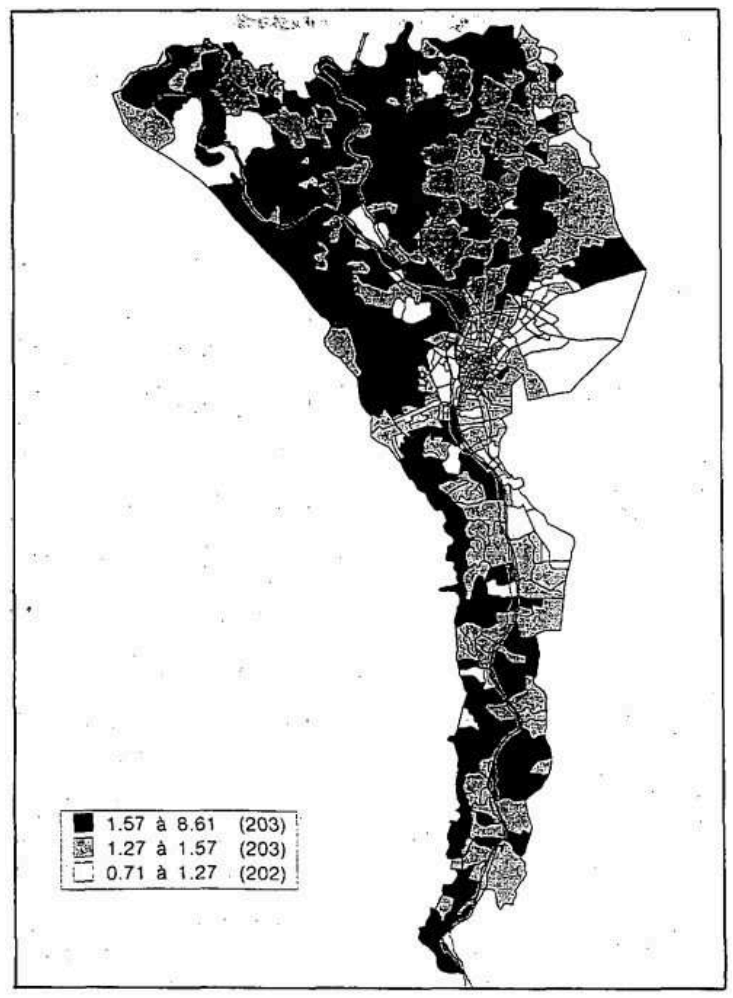

31 Ces jeunes générations de la décohabitation qui peuplent les périphéries sont des citadins de naissance. Leurs parents, demeurés au centre de l'agglomération, ne 
l'étaient pas toujours car ils appartenaient aux générations de l'exode rural. C'est ainsi que l'image de banlieues alimentées par l'exode rural, volontiers brossée par des médias hâtifs, ne restitue qu'une face de la réalité. A l'attraction sur l'environnement rural, qui n'a pas totalement disparu, se juxtapose déjà et se substitue progressivement un mécanisme de desserrement et de " dédensification » qui s'apparente plutôt au BigBang. La face cachée de la réalité, peut-être déjà prédominante dans une couronne rapprochée et certainement appelée à le devenir prochainement dans des couronnes plus éloignées, pourrait ainsi s'énoncer par ce qui revêt l'allure d'un paradoxe, tant les idées reçues sont ancrées : les banlieues tendent à être plus citadines que les centres.

Nées en ville, les générations de la décohabitation avaient par ailleurs, avant de se déplacer, poursuivi leur scolarité plus loin que leurs parents. Leur installation en banlieue va ainsi élever le niveau moyen d'éducation de celle-ci et abaisser celui du centre qu'ils quittent. La carte du niveau d'éducation fait certes apparaître un net désavantage des périphéries par rapport au centre. Mais ce désavantage résulte en partie d'un double masquage : les nouveaux arrivants voisinent avec une population de souche, rurale et moins instruite, et ils sont au début de leur cycle de vie, c'est-à-dire que leurs enfants n'ont pas encore achevé leur scolarité. Plus parlante est donc la carte de l'élévation du niveau d'instruction entre 1976 et 1986, qui désigne sans ambiguïté la progression des banlieues ainsi que la stagnation - par endroits une régression - qui en résulte au centre.

C'est ainsi qu'en se poursuivant, l'urbanisation change de forme et de contenu. Elle se fait toujours par adjonction de nouvelles couronnes, mais avec la substitution graduelle d'un mouvement centrifuge au mouvement centripète d'hier. Le premier accentuait la segmentation de l'espace, car les nouveaux arrivés ne ressemblaient pas à ceux au pourtour desquels ils s'établissaient, tandis que le second tend au lissage social. Les périphéries, qui concentraient dans les décennies passées une population en transition vers la modernité, tendent à concentrer aujourd'hui le produit de la modernité. Tout cela bien sûr n'est qu'une tendance, à peine perceptible. Mais le rôle des sciences sociales est aussi, en identifiant les changements qui se profilent aux marges, de sortir certains débats des ornières où le goût du drame les enlise.

\section{NOTES}

1. Communication présentée au colloque La société urbaine dans le monde arabe ; transformations, enjeux, perspectives, Fondation Giovanni Agnelli, Turin, 12-13/12/1994.

2. Les travaux du groupe d'experts sur la répartition de la population et les migrations, réuni à New-York par les Nations Unies dans le cadre des préparatifs de fond de la Conférence du Caire, révèlent fort bien ce nouvel état d'esprit, moins pessimiste qu'auparavant. Voir Bulletin démographique des Nations Unies n³4/35, New-York, 1994, pp. 143-179. 
3. L'Union internationale pour l'étude scientifique de la population, qui tient, depuis 1927 , un congrès tous les 4 ans et représente un lieu central de la construction disciplinaire de la démographie.

4. De E. Durkheim (De la division du travail social) à E. Boserup (The Conditions of Agricultural Progress, Allen \& Unwin, Londres, 1965), de nombreux auteurs ont souligné les bénéfices sociaux et économiques de la densité démographique : solidarité « organique » et spécialisation des tâches, économies d'échelle, progrès technique et innovation sous l'effet de la contrainte, etc.

5. Voir Pumain D., « Les systèmes de villes », Encyclopédie de géographie, Economica, Paris, 1992.

6. Wagih A., « La croissance du bâti dans la région du Grand Caire », Lettre de l'OUCC n ${ }^{\circ}$ 37, Cedej, Le Caire, 1994, pp. 24-30.

7. On ne dispose pas d'étude démographique récente sur la Libye.

8. Pour une vue globale des contrastes urbains dans le monde arabe, je me permets de renvoyer à R. Boustani et P. Fargues, Atlas du monde arabe. Géopolitique et société, Bordas, Paris, 1990 ; pour les comparaisons mondiales, à United Nations, World Urbanization Prospects : The 1992 Revision. Estimates and Projections of Urban and Rural Populations and of Rural Agglomerations, ST/ESA/SER.A 136, New-York, 1993.

9. Je reprends dans cette partie des arguments que j'ai tour à tour développés dans quatre publications ; «Explosion démographique ou rupture sociale », in Gh. Salamé (sous la direction de), Démocraties sans démocrates ?, Fayard, Paris, 1994 ; «Changing Hierarchies of Gender and Generation in the Arab World ", in C. Makhlouf (ed), Family, Gender and Population Policy in the Middle-East, Columbia University Press, 1995 (à paraître) ; « Note sur la diffusion de l'instruction scolaire en Égypte d'après les recensements ", Égypte/Monde arabe $\mathrm{n}^{\circ} 18-19,2^{\mathrm{e}}$ et $3^{\mathrm{e}}$ trim.1994, Cedej, et « Violence politique et démographie en Égypte ", in Le phénomène de la violence politique : perspectives comparatistes et paradigme égyptien, Cedej, Le Caire, 1994.

10. Le mot est emprunté à H. Sharabi, Neopatriarchy : a Theory of Distorted Change in Arab Society, Oxford University Press, Oxford \& New-York, 1988.

11. Voir R. Tabbarah, « Human Resources Development and its Population Dimension in the Arab World », Population Bulletin of the ESCWA n 32, Bagdad, 1988.

12. L'expression est de M. Chatelus, « Le monde arabe vingt ans après ", MaghrebMachrek n ${ }^{0}$ 101, Paris, 1983.

13. Sur l'activité économique des femmes en milieu populaire urbain, voir le remarquable travail de N. Fergany, Urban Women. Work and Poverty Alleviation in Egypt, Al-Mishkat, Le Caire, 1994.

14. Cette dernière partie présente des hypothèses que commencent à étayer les travaux en cours à l'Observatoire urbain du Caire contemporain (Cedej).

15. Dénomination non conforme à l'usage administratif, qui renvoie à une zone allant de Benha (non comprise) au nord, à al-Saff au sud.

16. Ce solde n'est pas donné par le recensement. Nous l'avons calculé dans chaque unité spatiale élémentaire i par différence entre le taux d'accroissement total et le taux d'accroissement naturel, ce dernier étant estimé comme suit : $\operatorname{TAN}_{\mathrm{i}}=\left[0,2 \mathrm{P}_{\mathrm{i}}(0,5) / \mathrm{S}\right] /\left[\mathrm{P}_{\mathrm{i}}\right.$

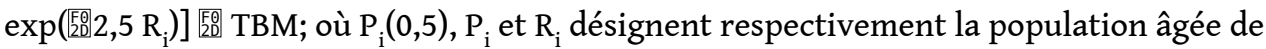
0-5 ans, la population totale et le taux d'accroissement de l'unité i. La probabilité de survie de la naissance jusqu'à l'âge de 0-5 ans, $\mathrm{S}$, et le taux brut de mortalité, TBM, sont uniformément fixés à 0,9 et 0,008 respectivement. 
INDEX

Mots-clés : démographie, monde arabe, urbanisation, ville

\section{AUTEUR}

PHILIPPE FARGUES

CEDEJ 